\title{
Dealing with COVID-19: initial perspectives of a small radiology department
}

Shawn Shi Xian $\underline{K o k}^{1}$, MBBS, FRCR, Mohammad Taufik Bin Mohamed Shah ${ }^{1}$, MBBS, FRCR, Wei Kiong $\underline{\text { Cheong }}^{1}$, MBBS, FRCR, Arthur Kim Chai Cheng ${ }^{1}$, MSc, Li Hoon Sng ${ }^{1}$, MMedRadSc, Parag Ratnakar Salkade ${ }^{1}$, MBBS, FRCR,

Steven Bak Siew Wong $^{1}$, MBChB, MMed

\section{INTRODUCTION}

Coronavirus disease 2019 (COVID-19) has taken the world by storm since a team from the Chinese Center for Disease Control and Prevention isolated a novel coronavirus from a cluster of patients with pneumonia of unknown aetiology in the city of Wuhan, Hubei Province, China. ${ }^{(1)}$ Shortly after, Singapore confirmed its first imported case on 23 January 2020. The World Health Organization declared COVID-19 an international public health emergency on 31 January 2020, and Singapore's alert level was raised to Disease Outbreak Response System Condition (DORSCON) Orange on 7 February 2020.(2)

The periodic threat of infectious disease outbreaks, while potentially debilitating to health systems, is not new to our island state, not least to radiology departments. The integral role of radiology in a pandemic has been well described historically as far back as the 1918 influenza pandemic, but perhaps none closer to home than the severe acute respiratory syndrome (SARS) outbreak in 2003 when radiology departments had to rapidly make operational changes. ${ }^{(3,4)}$

The Department of Radiology in Sengkang General Hospital is in its infancy, having been in official operation for just over 17 months at the time of the outbreak. We are also a young team, with most of our staff not yet in clinical practice during the SARS outbreak. Nonetheless, we have responded swiftly in implementing changes, cognisant of the need to abide by several core principles.

\section{STRIVING FOR NEAR NORMALCY IN BUSINESS CONTINUITY}

Striving to maintain normalcy meant continuing to provide timely imaging services and reports to our patients and clinician colleagues. Although outpatient 'no-show' rates in our department were gradually climbing, we remained committed to ensuring that semi-urgent outpatients are scanned on a timely basis, such as staging scans for oncology patients and computed tomography (CT) coronary angiography for patients with unstable angina.

Shortly after the local outbreak, a ministry-initiated 'SwabChest Radiograph (CXR)-Go' screening process was launched. The requirement for round-the-clock reporting of CXR with a shorter turnaround time of less than one hour presented a new operational challenge to our small department. While not requested by the ministry, we mandated that all screening CXR would be read by associate consultant-grade radiologists and above. This was partly due to the special circumstance of our dearth of senior residents/ service registrars at the current juncture.

Being a small team (comprising 17 diagnostic radiologists, two vascular and interventional radiologists and eight junior residents/ medical officers) in comparison to our counterparts in the larger restructured hospitals, the department would be particularly vulnerable in the event of an outbreak. Consequently, we divided the department into four organic teams comprising members of varying subspecialty capability and seniority. Teams are segregated into various reporting centres in the department and forbidden from contact, even during lunch hours. The most senior radiologist of each team is designated as the team leader. Taking into account the relatively small staff strength, geographical separation of the radiologists was deemed more appropriate than temporal separation. Additional reporting workstations were redeployed to several locations around the department to enable this. The arrangement was made possible by incorporating into the design of the department at the planning phase the need to have extra picture archiving and communication systems (PACS) network points for emergent use.

More manpower has also been allocated to 'after hours' services to address the need for shorter CXR turnaround time. This required the creative employment of a staggered shift system and a designated 'on-call' overnight radiologist to reduce manpower fatigue. Each weekday, a 'shift' radiologist is rostered from $1.30 \mathrm{pm}$ to $10.30 \mathrm{pm}$. On weekends, three radiologists are rostered in staggered four-hour shifts from $8.30 \mathrm{am}$ to $8.30 \mathrm{pm}$, with an 'on-call' radiologist covering overnight duties.

Radiographers are also separated into teams by location. This ensures that different teams are dedicated to serving inpatients, outpatients or emergency department (ED) patients. This is quite easily achievable in modalities such as general radiography, in which in- and outpatient areas are clearly demarcated by different sections, while emergency services are located within the ED on a separate level. Interventional radiology and cardiology sections are already geographically separated from the main diagnostic sections of the department, further reducing potential cross-contamination.

Within the ED, radiology services are further segregated into P1, P2, P3 and isolation, and each section is equipped with its

${ }_{1}^{1}$ Department of Radiology, Sengkang General Hospital, Singapore

Correspondence: Dr Shawn Kok Shi Xian, Associate Consultant, Department of Radiology, Sengkang General Hospital, 110 Sengkang East Way, Singapore 544886. shawn.kok.s.x@singhealth.com.sg 
own static general radiographic units. Mobile digital radiographic units were redeployed from inpatient use to the ED to ensure minimal movement of patients requiring CXR. A total of 20 radiographers are split into five teams to manage the general radiography load at the ED. Each team has an assigned team leader who is the main point of contact for any new updates on operational issues. A designated radiographer on rotation at the isolation area provides general radiographic services to suspect cases. This radiographer dons full personal protective equipment (PPE) when attending to such patients, and performs a complete wipe-down of the equipment after every case.

Additionally, there is a pre-designated 'dirty room' for advanced modalities such as $\mathrm{CT}$ and magnetic resonance imaging to manage any suspected or confirmed COVID-19 case. Once utilised, terminal cleaning is activated, and it typically takes up to 60 minutes before the room is deemed ready to receive the next case.

\section{MAINTAINING STAFF MORALE AND SAFETY}

With team segregation and a staggered shift system, coupled with an underlying fear of a disease yet to be fully understood, staff members inadvertently have to step out of their comfort zones. This required a shift in mindset and a certain public sector ethos. Regular and clear communication with staff at all levels is critical to avoid declining morale, as staff prefer to be 'kept in the loop' ${ }^{(5)}$ Even as senior radiologists and radiographers attended hospital-level meetings and contemplated directives, small intra-departmental committees were formed to facilitate brainstorming of ideas and dissemination of information to the ground. Secure messaging applications such as 'Tiger Connect' greatly aid in this respect.

The radiology department also engages staff in communication sessions with the department head, senior manager and senior hospital leaders so that they are kept informed of the evolving situation. Team leaders play a vital role in ensuring conformity to safe practices, such as reminding staff to perform daily temperature taking, ensuring correct donning of appropriate PPE, organising refresher courses on powered air-purifying respirators, and retesting of mask fitting to ensure everyone is adequately protected.

Donning PPE and working on the frontline is a challenge for all healthcare workers, not least our ED radiographers. ${ }^{(6)}$ Nursing staff, particularly those in the vascular and interventional radiology team, are also stretched thin, as more manpower is required to ensure strict enforcement of pre- and post-procedure infection control protocols. Team members are constantly reminded to look for signs of stress among colleagues and to promptly raise red flags to team leaders.

\section{CONTINUING OUR EDUCATIONAL MISSION - LEVERAGING ON TECHNOLOGY}

Besides clinical duties and responsibilities, the department also contributes to undergraduate education (radiography and medical students) and postgraduate professional development and training (residents, medical officers and nurses). It also collaborates with other departments in institutional educational activities for the purpose of continued clinical service provision (e.g. clinical-radiological conferences) and continuing professional development (e.g. allied health interprofessional rounds).

Over and above department-specific interventions, additional mitigation measures have been imposed by external stakeholders at both the institutional and national levels (e.g. resident training committees, public universities and private education providers), which were aimed at preventing cross-cluster and cross-institution spread of disease among healthcare professionals. This presented new challenges in ensuring continuing professional development. As our residents are at a relatively novice stage of specialty training, we were concerned that segregation into even smaller subgroups, where educational and training needs can differ significantly, could compromise their educational experience.

We took this opportunity to explore technological adjuncts that permit remote and synchronous team learning and multidisciplinary collaborative efforts. In our institution, cloud-based video-conferencing using Zoom (Zoom Video Communications Inc, San Jose, CA, USA) is an example of a computer-supported collaborative work application that has been successfully adopted for clinico-radiological rounds and interprofessional education. The platform allows for secure recording and storage of sessions without the use of third-party software applications, features that are vital for protection of highly sensitive data. ${ }^{(7)}$

Technology also facilitates teaching and learning for residents and medical officers. Our PACS workstation promotes synchronous interactions by enabling the sharing of the image viewer interface using the local hospital network, allowing for immediate faculty feedback to learners or opportunistic just-intime learning. ${ }^{(8)}$ As such, we are able to deliver blended learning activities while retaining the interactive and collaborative environment of face-to-face tutorials. One platform that we have successfully employed with positive feedback is Webex (http://www.Webex.com), with interactive tutorial sessions enhanced by the platform's features such as online whiteboards to illustrate concepts, availability of an audience response system for evaluation of learning, and ability to share supplementary learning resources, securely record and store teaching sessions for future review. ${ }^{(9)}$

\section{CONCLUSION}

Finally, it should be said that although the threat of COVID-19 is far from over and continued vigilance is paramount, every crisis presents an opportunity to challenge and refine our operational practices. As the situation evolves, departments should adopt regular reflective reviews of their processes, discarding negative practices and retaining good ones even as we look forward to a sense of normalcy post COVID-19. Collaborative research efforts with our clinical colleagues have not been hampered by hospital- or cluster-level segregation as we harness technology to continue our efforts to better understand and battle the disease. ${ }^{(6)}$ 


\section{REFERENCES}

1. Zhu N, Zhang D, Wang $W$, et al. A novel coronavirus from patients with pneumonia in China, 2019. N Engl J Med 2020; 382:727-33.

2. Lum LHW, Tambyah PA. Outbreak of COVID-19 - an urgent need for good science to silence our fears? Singapore Med J 2020; 61:55-7.

3. Mollura DJ, Morens DM, Taubenberger JK, Bray M. The role of radiology in influenza: novel H1N1 and lessons learned from the 1918 pandemic. J Am Coll Radiol 2010; 7:690-7.

4. Tsou IY, Goh JS, Kaw GJ, Chee TS. Severe acute respiratory syndrome: management and reconfiguration of a radiology department in an infectious disease situation. Radiology 2003; 229:21-6.

5. Odine M. Communication problems in management. J Emerg Issues Econ
Finance Banking 2015; 4:1615-30.

6. Wong JEL, Leo YS, Tan CC. COVID-19 in Singapore-current experience: critical global issues that require attention and action. JAMA 2020 Feb 20. https://doi. org/10.1001/jama.2020.2467. [Epub ahead of print]

7. Archibald MM, Ambagtsheer RC, Casey MG, Lawless M. Using Zoom videoconferencing for qualitative data collection: perceptions and experiences of researchers and participants. Int J Qual Methods 2019; 18. https://doi. org/10.1177/1609406919874596

8. Eng J, Leal JP, Shu W, Yang GL. Collaboration system for radiology workstations. Radiographics 2002; 22:e5.

9. Woods M, Rosenberg ME. Educational tools: thinking outside the box. Clin I Am Soc Nephrol 2016; 11:518-26. 